NUMBER THEORY WEEK 2017

BANACH CENTER PUBLICATIONS, VOLUME 118

INSTITUTE OF MATHEMATICS

POLISH ACADEMY OF SCIENCES

WARSZAWA 2019

\title{
THE MODULAR DISTRIBUTION OF STERN'S SEQUENCE
}

\author{
JEAN-MARC DESHOUILLERS \\ Institut Mathématique de Bordeaux, Université de Bordeaux \\ Bordeaux INP and CNRS, 33405 Talence, France \\ E-mail: jean-marc.deshouillers@math.u-bordeaux.fr \\ ANDRZEJ SCHINZEL \\ Institute of Mathematics, Polish Academy of Sciences \\ Śniadeckich 8, 00-656 Warszawa, Poland \\ E-mail: schinzel@impan.pl
}

To our Friend and Colleague Jerzy Kaczorowski, for his 60th anniversary

Abstract. Let $\left(s_{n}\right)_{n}$ be Stern's sequence, $a, b, m>0$ integers. The natural density of indices $n$ such that $\left(s_{n}, s_{n+1}\right) \equiv(a, b) \bmod m$ exists and is determined. The main tools in the proof are the properties of the relevant automata.

1. Introduction. Stern [7 defined in 1858 the following sequence

$$
s_{0}=0, \quad s_{1}=1 \quad \text { and, for } n \geq 1: \quad s_{2 n}=s_{n}, s_{2 n+1}=s_{n}+s_{n+1} .
$$

The paper [5] presents the main properties of this sequence.

Klavžar, Milutinović and Petr [4] defined similarly the sequence of Stern polynomials:

$$
\begin{aligned}
& B_{0}(x)=0, \quad B_{1}(x)=1, \\
& \text { and, for } n \geq 1: B_{2 n}(x)=x B_{n}(x), B_{2 n+1}(x)=B_{n}(x)+B_{n+1}(x) .
\end{aligned}
$$

Ulas 8 conjectured that the only rational zeroes of $B_{n}(x)$ are $0,-1,-1 / 2,-1 / 3$. This conjecture was proved by Gawron [3] who showed that the lower density of the sequences of indices $n$ such that $B_{n}(-1 / 2)=0$ or such that $B_{n}(-1 / 3)=0$ is 0 , and conjectured that

2010 Mathematics Subject Classification: Primary 11B37, 11B85.

Key words and phrases: Stern's sequence, deterministic finite automata.

The paper is in final form and no version of it will be published elsewhere. 
the density actually exists, and is equal to 0 . This conjecture was proved by the second named author [6] who showed that for $\nu \in\{2,3\}$ there is an infinite sequence of primes $p_{n}^{(\nu)}$ such that the upper density of the sequence of indices $n$ such that $B_{n}(-1 / \nu) \equiv 0 \bmod p_{n}^{(\nu)}$ exists and tends to 0 when the prime $p_{n}^{(\nu)}$ tends to infinity. He also observed that for any prime $p$ the upper density of the sequence of indices $n$ such that $s_{n} \equiv 0 \bmod p$ does not exceed $2 / p$. This is improved in the following results.

TheOREM 1.1. For any positive integer $m$ and any pair $(a, b)$ of rational integers, we have

$$
\begin{aligned}
\lim _{x \rightarrow \infty} \frac{1}{x} \operatorname{Card}\left\{n \leq x:\left(s_{n}, s_{n+1}\right) \equiv\right. & (a, b) \bmod m\} \\
& = \begin{cases}\frac{1}{m^{2}} \prod_{p \mid m}\left(1-\frac{1}{p^{2}}\right)^{-1} & \text { if } \operatorname{gcd}(a, b, m)=1 \\
0 & \text { otherwise, }\end{cases}
\end{aligned}
$$

where the product is taken over the prime factors $p$ of $m$.

The first corollary of this result was proposed by the second named author during the Number Theory Week held in Poznań in September 2017, and proved by him for $p<10$.

COROllary 1.2. For any prime $p$ and any residue $r$ modulo $p$, the natural density of the sequence of indices $n$ such that

$$
s_{n} \equiv r s_{n+1} \bmod p
$$

exists and is equal to $1 /(p+1)$.

COROLlaRY 1.3. For any positive integer $m$ and any rational integer a, the sequence of indices $n$ such that $s(n) \equiv a \bmod m$ has a natural density which is equal to

$$
\frac{1}{m} \prod_{\substack{p|m \\ p| a}}\left(1+\frac{1}{p}\right)^{-1} \prod_{\substack{p \mid m \\ p \nmid a}}\left(1-\frac{1}{p^{2}}\right)^{-1} .
$$

A 2-automatic structure associated to the sequence of the Stern polynomials has been noticed by Gawron [3. It turns out that the relevant automata have two specific properties, which are easy to check and imply the existence and the value of the natural density of the sequences they generate, a point which seems to have been overlooked in the previous papers on the subject. We thus devote the next section to the study of such automata. We shall give in the last section the proof of the results which we have just stated.

2. The $b$ - $I$-automata. We describe here the deterministic finite automata which are of interest for this paper and give the properties we use; we shall call b-I-automata (or simply I-automata) those automata. As much as we can, we refer to the Allouche-Shallit monograph [1]. We first give their formal definition, which follows pp. 128-129 of [1], then explain how they are used for generating sequences of integers, as in Chapter 5 of [1]; we then give the main property of sequences generated by $I$-automata and finally prove them, using the consequence of the Perron-Frobenius theorem given in Chapter 8 of [1]. 
The main result of this section thus combines well-known facts, but it seems convenient to state it independently of the precise context in which we are using it.

Definition 2.1. A $b$ - $I$-automaton is defined by a 5 -tuple $\mathcal{A}=\left(Q, \Sigma, \delta, q_{0}, F\right)$, where

- $Q$ is a non-empty set (the set of the states),

- $\Sigma=\{0,1, \ldots, b-1\}$, where $b \geq 2$ (the alphabet),

- $\delta: Q \times \Sigma \rightarrow Q$ (the transition function),

- $q_{0} \in Q$ (the initial state),

- $F \subset Q$ (the accepting states),

satisfying the following two properties

(i) $\delta\left(q_{0}, 0\right)=q_{0}$,

(ii) for any $e \in \Sigma$, the $\operatorname{map} \delta(\cdot, e)$ from $Q$ to $Q$ is invertible.

Condition (ii) was not considered in [1. M. Drmota and J. Morgenbesser [2] call invertible an automaton satisfying Definition 2.1 with a stronger condition than (i), namely: for any $q$ in $Q$, one has $\delta(q, 0)=q$.

As in [1, p. 129, we extend the application $\delta$ to an application, still denoted as $\delta$, from the set $\Sigma^{*}$ of the words over $\Sigma$ to $Q$ satisfying $\delta(q, \emptyset)=q$ for any $q \in Q$ and $\delta(q, x a)=\delta(\delta(q, x), a)$ for any $q \in Q, x \in \Sigma^{*}$ and $a \in \Sigma$.

In our case, the set $\Sigma$ can be understood as the digits used for representing an integer in the base $b$ : with a word $x=e_{k} e_{k-1} \cdots e_{0} \in \Sigma^{*}$ we can associate the number $n(w)=$ $\sum_{\ell=0}^{k} e_{\ell} b^{\ell}$. In the other way round, there are several ways to associate to a non-negative integer $n$ a word in $\Sigma^{*}$. However, one of the merits of condition (i) is that if $x_{1}$ and $x_{2}$ are two words associated to $n$, we have $\delta\left(q_{0}, x_{1}\right)=\delta\left(q_{0}, x_{2}\right)$, since $x_{1}$ and $x_{2}$ may at most differ by the number of 0 's on their left. Thus, the representation used for $n$ is not relevant; for convenience we shall choose $[n]$ to represent the shortest representation of $n$ in base $b$.

Let $\mathcal{A}$ as above be an $I$-automaton. Following Chapter 5 of [1], we say that the set $\mathcal{T} \subset \mathbb{N}$ is generated by $\mathcal{A}$ if

$$
n \in \mathcal{T} \Leftrightarrow \delta\left(q_{0},[n]\right) \in F .
$$

We say that a set $\mathcal{T} \subset \mathbb{N}$ has an natural density or simply a density (in [1] the expression frequency is used), if the limit

$$
\lim _{N \rightarrow \infty} \frac{1}{N} \operatorname{Card}\{n \leq N: n \in \mathcal{T}\}
$$

exists. When it does, this limit is called the natural density (or density) of $\mathcal{T}$. Finally, following [1], p. 129, we say that a state $q \in Q$ is reachable if there exists an $x \in \Sigma^{*}$ such that $q=\delta\left(q_{0}, x\right)$, and unreachable otherwise. We are now in a position to state the main result of this section.

THEOREM 2.2. Let $\mathcal{A}=\left(Q, \Sigma, \delta, q_{0}, F\right)$ be an I-automaton. The natural density of the set of integers generated by $\mathcal{A}$ exists and is equal to the quotient of the number of the reachable states in $F$ by the number of the reachable states in $Q$.

Proof. If $F$ is the disjoint union of $F_{1}$ and $F_{2}$, the sequence generated by $\left(Q, \Sigma, \delta, q_{0}, F\right)$ is the disjoint union of the sequences generated by $\left(Q, \Sigma, \delta, q_{0}, F_{1}\right)$ and $\left(Q, \Sigma, \delta, q_{0}, F_{2}\right)$ and 
the number of reachable elements of $F$ is the sum of the number of reachable elements of $F_{1}$ and $F_{2}$; thus if the theorem is valid for $F_{1}$ and $F_{2}$, it is valid for $F$. It is thus enough to prove it when $F$ has only one element. Moreover, the result is trivially true when $F=\{q\}$, where $q$ is unreachable. It is thus enough to prove the theorem when $F=\{q\}$, where $q$ is reachable. Moreover, without loss of generality, we may assume that all the elements of $Q$ are reachable. That is what we assume in the sequel.

We index the elements of $Q$ (the indexation has no importance except that we keep the notation $q_{0}$ for the initial state) and write $Q=\left\{q_{0}, q_{1}, \ldots, q_{K-1}\right\}$, where $K=\operatorname{Card}(Q)$. For each digit $\ell \in \Sigma$, we define the matrix $M_{\ell}$ such that its entry at the intersection of the $i$-th row and the $j$-th column is 1 if $\delta\left(q_{j}, \ell\right)=q_{i}$ and 0 otherwise. Condition (ii) is equivalent to the fact that for each $\ell$ the matrix $M_{\ell}$ is a permutation matrix. The matrix $M=\sum_{\ell=0}^{b-1} M_{\ell}$ is called the incidence matrix (cf. [1], p. 248).

An important property of the incidence matrices associated to $I$-automata is the following lemma.

LEMma 2.3. The incidence matrix $M$ of any I-automaton is primitive, which means that there exists an integer $k$ such that $M^{k}$ has only positive entries.

Proof. We borrow the terminology primitive from [1] p. 250; notice that this definition differs from that used in several standard books. The lemma is equivalent to saying that there exists an integer $k$ such that for any $i$ and $j$ in $\{0,1, \ldots, K-1\}$, there exists a word $x \in \Sigma^{*}$ of length $k$ such that $\delta\left(q_{j}, x\right)=q_{i}$. This is a simple consequence of the following three points.

(A) For any $i \in\{0,1, \ldots, K-1\}$, there exists a word $x_{i}$ such that $\delta\left(q_{0}, x_{i}\right)=q_{i}$. This is just a way of saying that $q_{i}$ is reachable, which we assumed.

(B) For any non-negative $m$, we have $\delta\left(q_{0}, 0^{(m)}\right)=q_{0}$, where $0^{(m)}$ denotes the word $000 \cdots 000$ consisting of $m$ consecutive zeroes. This is a trivial consequence of the property (i) stating that $\delta\left(q_{0}, 0\right)=q_{0}$.

(C) For any $j \in\{0,1, \ldots, K-1\}$, there exists a word $y_{j}$ such that $\delta\left(q_{j}, y_{j}\right)=q_{0}$. By (A), there exists a word $x_{j}=e_{r} e_{r-1} \cdots e_{2} e_{1}$ such that $q_{j}=\delta\left(q_{0}, x_{j}\right)$; we claim that the word

$$
z_{j}=e_{1}^{(K !-1)} e_{2}^{(K !-1)} \cdots e_{r-1}^{(K !-1)} e_{r}^{(K !-1)}
$$

is an admissible word for $y_{j}$. Let us compute $\delta\left(q_{j}, z_{j}\right)$; we have

$$
\begin{aligned}
\delta\left(q_{j}, z_{j}\right) & =\delta\left(\delta\left(q_{0}, x_{j}\right), z_{j}\right)=\delta\left(q_{0}, x_{j} z_{j}\right) \\
& =\delta\left(q_{0},\left(e_{r} e_{r-1} \cdots e_{2} e_{1}\right)\left(e_{1}^{(K !-1)} e_{2}^{(K !-1)} \cdots e_{r-1}^{(K !-1)} e_{r}^{(K !-1)}\right)\right) \\
& =\delta\left(q_{0},\left(e_{r} e_{r-1} \cdots e_{2}\right)\left(e_{1} e_{1}^{(K !-1)}\right)\left(e_{2}^{(K !-1)} \cdots e_{r-1}^{(K !-1)} e_{r}^{(K !-1)}\right)\right) \\
& =\delta\left(\delta\left(\delta\left(q_{0}, e_{r} \cdots e_{2}\right), e_{1}^{(K !)}\right), e_{2}^{(K !-1)} \cdots e_{r}^{(K !-1)}\right) .
\end{aligned}
$$

The key point to notice here is that condition (ii) which says that for any $e$ in $\Sigma$, the map $\delta(\cdot, e)$ is invertible, implies that its order is a divisor of that of the permutation group $\mathfrak{S}_{K}$ and thus $\delta\left(q, e^{(K !)}\right)$ is equal to $q$ for any state $q$ and any digit $e$. We thus have by induction

$$
\delta\left(q_{j}, z_{j}\right)=\delta\left(\delta\left(q_{0}, e_{r} \cdots e_{2}\right), e_{2}^{(K !-1)} \cdots e_{r}^{(K !-1)}\right)=\ldots=\delta\left(q_{0}, \emptyset\right)=q_{0} .
$$


Equipped with (A), (B) and (C), let us finish the proof of Lemma 2.3. By Property (A), we can go from $q_{0}$ to any $q_{i}$ in a finite number of steps (i.e. using words of finite lengths); this finite set of finite numbers is bounded, say by $K_{1}$ (it is indeed easy to see that $K$ is a suitable bound, but we do not need this precision); the same applies to the number of steps to go from any $q_{i}$ to $q_{0}$, which is bounded by some $K_{2}$ (again, $K$ is admissible). Now, we see that we can always go from any $q_{j}$ to any $q_{i}$ in exactly $K_{1}+K_{2}$ steps by going from $q_{j}$ to $q_{0}$, stepping on $q_{0}$ a suitable number of times, and going from $q_{0}$ to $q_{i}$. Thus $K_{1}+K_{2}$ (which can be taken to be equal to $2 K$ ) is a suitable value for the $k$ announced in the statement of Lemma 2.3.

We come back to the proof of Theorem 2.2. Once we know that the incidence matrix $M$ is primitive, Theorem 8.4.7 of [1] tells us that the natural density of the sequence generated by our $I$-automaton $\left(Q, \Sigma, \delta, q_{0},\{q\}\right)$ exists. Moreover, the vector of densities of the sequences generated by the automaton $\left(Q, \Sigma, \delta, q_{0},\left\{q_{i}\right\}\right)$ is proportional to the Perron-Frobenius vector of the incidence matrix. Because of (ii), the sum of all the entries of any row from $M$ are equal to $b$. This implies that the vector ${ }^{t}(1,1, \ldots, 1,1)$ is a Perron-Frobenius vector of $M$, and thus, the natural densities of all the sequences generated by the automaton $\left(Q, \Sigma, \delta, q_{0},\left\{q_{i}\right\}\right)$ are equal. This implies that their common natural density is $1 / \operatorname{Card}(Q)$, which proves Theorem 2.2

3. Proof of the main results. In this section, we consider a positive integer $m$ and for a rational integer $x$, we shall denote by $\bar{x}$ the class of $x$ modulo $m$. For proving Theorem 1.1. we shall describe a 2 - $I$-automaton which generates the sequence $\left(\overline{s_{n}}, \overline{s_{n+1}}\right)_{n}$ and study the set of its reachable states.

We introduce two maps $U_{0}$ and $U_{1}$ from $(\mathbb{Z} / m \mathbb{Z})^{2}$ to $(\mathbb{Z} / m \mathbb{Z})^{2}$ defined by

$$
U_{0}(\bar{x}, \bar{y})=(\bar{x}, \bar{x}+\bar{y}), \quad U_{1}(\bar{x}, \bar{y})=(\bar{x}+\bar{y}, \bar{y}) .
$$

Relation (1), read modulo $m$, leads to

$$
\overline{s_{0}}=\overline{0}, \quad \overline{s_{1}}=\overline{1} \quad \text { and, for } n \geq 1: \overline{s_{2 n}}=\overline{s_{n}}, \overline{s_{2 n+1}}=\overline{s_{n}}+\overline{s_{n+1}} .
$$

and a short computation leads to

$$
\left(\overline{s_{2 n}}, \overline{s_{2 n+1}}\right)=\left(\overline{s_{n}}, \overline{s_{n}}+\overline{s_{n+1}}\right)=U_{0}\left(\overline{s_{n}}, \overline{s_{n+1}}\right)
$$

and

$$
\left(\overline{s_{2 n+1}}, \overline{s_{2 n+2}}\right)=\left(\overline{s_{n}}+\overline{s_{n+1}}, \overline{s_{n+1}}\right)=U_{1}\left(\overline{s_{n}}, \overline{s_{n+1}}\right) .
$$

Proposition 3.1. Let $a$ and $b$ be two rational integers. The sequence of the integers $n$ such that $\left(\overline{s_{n}}, \overline{s_{n+1}}\right)=(\bar{a}, \bar{b})$ is generated by the 2 -I-automaton

$$
\mathcal{A}_{m}=\left((\mathbb{Z} / m \mathbb{Z})^{2},\{0,1\}, \delta_{m},(\overline{0}, \overline{1}),\{(\bar{a}, \bar{b})\}\right),
$$

where

$$
\forall q \in(\mathbb{Z} / m \mathbb{Z})^{2}: \delta_{m}(q, 0)=U_{0}(q) \text { and } \delta_{m}(q, 1)=U_{1}(q)
$$

Proof. All the pairs $\xi_{n}=\left(\overline{s_{n}}, \overline{s_{n+1}}\right)$ belong to $(\mathbb{Z} / m \mathbb{Z})^{2}$, which we take as the set of states. Relations (7) and (8) tell us that $\xi_{2 n}$ and $\xi_{2 n+1}$ depend on $\xi_{n}$, regardless of $n$, which justifies that we have a 2 -automaton (i.e. $\Sigma=\{0,1\}$ ) and the same relations tell us how they depend on $\xi_{n}$, which justifies the definition of $\delta$. We further have $\xi_{0}=\left(\overline{s_{0}}, \overline{s_{1}}\right)=$ $(\overline{0}, \overline{1})$, whence the choice of $q_{0}$. Finally, the choice of $F$ to be $\{(\bar{a}, \bar{b})\}$ corresponds to the 
fact that we wish to detect those integers $n$ for which $\left(\overline{s_{n}}, \overline{s_{n+1}}\right)=(\bar{a}, \bar{b})$. It remains to show that the automaton we have introduced is a 2 - $I$-automaton.

Condition (i) from Definition 2.1 comes simply from the fact that $U_{0}(\overline{0}, \overline{1})=(\overline{0}, \overline{1})$ and condition (ii) from the fact that $U_{0}$ and $U_{1}$ are invertible linear operators on $(\mathbb{Z} / m \mathbb{Z})^{2}$ (with respective inverses $U_{0}^{-1}$ and $U_{1}^{-1}$ defined by $U_{0}^{-1}(\bar{x}, \bar{y})=(\bar{x},-\bar{x}+\bar{y}), U_{1}^{-1}(\bar{x}, \bar{y})=$ $(\bar{x}-\bar{y}, \bar{y}))$.

We now determine the set $R_{m}$ of reachable states of the automaton $\mathcal{A}_{m}$.

Proposition 3.2. The reachable states of the automaton $\mathcal{A}_{m}$ introduced in Proposition 3.1 is the set

$$
R_{m}=\{(\bar{a}, \bar{b}): \operatorname{gcd}(a, b, m)=1\} .
$$

Proof. The fact that all reachable states $\left(\overline{s_{n}}, \overline{s_{n+1}}\right)$ are in $R_{m}$ is a direct consequence of the fact that for any integer $n \geq 0$, the consecutive Stern numbers $s_{n}$ and $s_{n+1}$ are coprime ([7, p. 199).

The proof of the reverse assertion (Lemma 3.4 below) will use the following result.

Lemma 3.3. Let $(a, b)$ be rational integers such that $\operatorname{gcd}(a, b, m)=1$. There exists a positive integer $k$ such that $\operatorname{gcd}(a-k b, m)=1$.

Proof. We let $k$ to be the greatest factor of $m$ which is coprime to $a$. Let $p$ be a prime factor of $m$.

If $p$ divides $a$, by assumption $p$ does not divide $b$ and by definition $p$ does not divide $k$ so that $p$ does not divide $a-k b$, and thus does not divide $\operatorname{gcd}(a-k b, m)$.

If $p$ does not divide $a$, by definition $p$ divides $k$, thus $p$ does not divide $a-k b$, and thus does not divide $\operatorname{gcd}(a-k b, m)$.

Proposition 3.2 will follow from the next lemma.

Lemma 3.4. For any pair $(a, b)$ of rational integers such that $\operatorname{gcd}(a, b, m)=1$, the pair $(\bar{a}, \bar{b})$ is reachable.

Proof. For any integer $k \geq 0$ we have $U_{1}^{k}(\overline{0}, \overline{1})=(\bar{k}, \overline{1})$ and $U_{0}^{k}(\overline{1}, \overline{1})=(\overline{1}, \overline{k+1})$, which gives us a first set of reachable elements.

Let $x$ be an integer coprime to $m$; we can find a positive integer $\ell$ such that $\ell x \equiv$ $-1 \bmod m$. If we apply $\ell$ times $U_{0}$ to $(\bar{x}, \overline{1})$, we obtain $(\bar{x}, \ell \bar{x}+\overline{1})=(\bar{x}, \overline{0})$ which is thus reachable.

Let $x$ be an integer coprime to $m$; then for any $y \in \mathbb{Z} / m \mathbb{Z},(\bar{x}, y)$ is reachable: indeed, since $(\bar{x}, \overline{0})$ is reachable, for any $h$, the element $U_{0}^{h}(x, \overline{0})=(\bar{x}, h \bar{x})$ is reachable, and since $\bar{x}$ is invertible in $\mathbb{Z} / m \mathbb{Z}$, the family $h \bar{x}$ covers $\mathbb{Z} / m \mathbb{Z}$.

Let finally $a$ and $b$ be two rational integers such that $\operatorname{gcd}(a, b, m)=1$. By Lemma 3.3 . there exists a positive integer $k$ such that $a-k b$ is coprime to $m$; we apply the previous claim with $x=a-k b$ and $y=\bar{b}$ : the state $(\bar{a}-k \bar{b}, \bar{b})$ is reachable, and so is $U_{1}^{k}(\bar{a}-k \bar{b}, \bar{b})$, which is equal to $(\bar{a}, \bar{b})$.

This ends the proof of Lemma 3.4 as well as that of Proposition 3.2

We now compute the cardinality of the set $R_{m}$ of reachable states of the automaton $\mathcal{A}_{m}$. 
Proposition 3.5. The number of reachable states of the automaton $\mathcal{A}_{m}$ introduced in Proposition 3.1 is

$$
\operatorname{Card}\left(R_{m}\right)=m^{2} \prod_{p \mid m}\left(1-\frac{1}{p^{2}}\right) .
$$

Proof. We use the characterization of the reachable states given by Proposition 3.2 . We have

$$
\begin{aligned}
\operatorname{Card}\left(R_{m}\right) & =\sum_{\substack{0 \leq a<m \\
0 \leq b<m \\
\operatorname{gcd}(a, b, m)=1}} 1=\sum_{d \mid m} \sum_{\substack{0 \leq a<m \\
\operatorname{gcd}(a, m)=d \operatorname{gcd}(b, d)=1}} \sum_{\substack{0 \leq b<m \\
\cos (a)}} 1 \\
& =m \sum_{d \mid m} \varphi\left(\frac{m}{d}\right) \frac{\varphi(d)}{d}=m \psi(m)
\end{aligned}
$$

where $\psi(m)$ is the Dirichlet convolution of two multiplicative functions. Thus $\psi(m)$ is a multiplicative function and it is sufficient to evaluate it on prime powers.

We have

$$
\psi(p)=(p-1) \cdot 1+1 \cdot\left(1-\frac{1}{p}\right)=p-\frac{1}{p}=p\left(1-\frac{1}{p^{2}}\right)
$$

and, for $k \geq 2$,

$$
\psi\left(p^{k}\right)=\left(p^{k-1}-p^{k-2}+\cdots+p-1\right)(1-1 / p)+\left(p^{k}-p^{k-1}\right)=p^{k}\left(1-1 / p^{2}\right) .
$$

The multiplicative function $\psi(m)$ coincide on all prime powers with the multiplicative function $m \prod_{p \mid m}\left(1-1 / p^{2}\right)$. Those two functions are thus equal and we have proved (10).

Proof of Theorem 1.1. Theorem 1.1 is a straightforward application of Theorem 2.2 which explains how to compute the density of a sequence generated by a $b$ - $I$-automaton in terms of the reachable states, Proposition 3.1. which states that the sequences under consideration in Theorem 1.1 are generated by a 2- $I$-automaton, Proposition 3.2 which describes the reachable states and Proposition 3.5. which counts those reachable states.

Proof of Corollary 1.2. In the case when $m=p$ is a prime number, all the states are reachable, except $(\overline{0}, \overline{0})$, which leads to a density of $1 /\left(p^{2}-1\right)$ for each reachable pair. Thus, for any $r$, the number of reachable pairs $(\bar{a}, \bar{b})$ such that $\bar{a}=r \bar{b}$ is $p-1$ and the density of the sequence of indices $n$ such that $s_{n} \equiv r s_{n+1} \bmod p$ is $(p-1) /\left(p^{2}-1\right)=1 /(p+1)$.

Proof of Corollary 1.3. We can either deduce Corollary 1.3 from our general result, or notice that the sequence of the integers $n$ such that $s(n) \equiv a \bmod m$ is a union of sequences, the natural densities of which exist and are given by Theorem 1.1. In either case, the result easily follows from the following lemma.

LEMma 3.6. For any rational integer a, we have

$$
\operatorname{Card}\{b \in[0, m-1]: \operatorname{gcd}(a, b, m)=1\}=m \prod_{\substack{p|m \\ p| a}}\left(1-\frac{1}{p}\right) .
$$


Proof. We have

$$
\begin{aligned}
\operatorname{Card}\{b \in[0, m-1]: \operatorname{gcd}(a, b, m)=1\} & =\sum_{\substack{0 \leq b<m \\
\operatorname{gcd}(b, \operatorname{gcd}(a, m))=1}} 1 \\
& =\frac{m}{\operatorname{gcd}(a, m)} \varphi(\operatorname{gcd}(a, m))=m \frac{\varphi(\operatorname{gcd}(a, m))}{\operatorname{gcd}(a, m))} \\
& =m \prod_{\substack{p|a \\
p| m}}\left(1-\frac{1}{p}\right) .
\end{aligned}
$$

Acknowledgments. Both authors highly appreciated the organization of the Poznań Number Theory Week 2017, which, among other, permitted them to start their collaboration on Stern's sequence.

The first-named author acknowledges the financial support of the Poznań Number Theory Week 2017, his research was also partially supported by the CEFIPRA project 5401-A and the MuDeRa FWF-ANR project.

\section{References}

[1] J-P. Allouche, J. Shallit, Automatic Sequences: Theory, Applications, Generalizations, Cambridge Univ. Press, Cambridge, 2003.

[2] M. Drmota, J. Morgenbesser, Generalized Thue-Morse sequences of squares, Israel J. Math. 190 (2012), 157-193.

[3] M. Gawron, A note on the arithmetic properties of the Stern polynomials, Publ. Math. Debrecen 85 (2014), 453-465.

[4] S. Klavžar, U. Milutinović, C. Petr, Stern polynomials, Adv. in Appl. Math. 39 (2007), 86-95.

[5] S. Northshield, Stern's diatomic sequence $0,1,1,2,1,3,2,3,1,4, \ldots$, Amer. Math. Monthly 117 (2010), 581-598.

[6] A. Schinzel, On the factors of Stern polynomials II. Proof of a conjecture of M. Gawron, Publ. Math. Debrecen 91 (2017), 515-524.

[7] M. A. Stern, Über eine zahlentheorische Funktion, J. Reine Angew. Math. 55 (1858), 193-220.

[8] M. Ulas, On certain arithmetic properties of Stern polynomials, Publ. Math. Debrecen 79 (2011), 55-81. 